\title{
The effects and prospects of the integration of traditional Chinese medicine and Western medicine on andrology in China
}

\author{
Wei-Guo Ma and Jin-Ming Jia
}

\begin{abstract}
Andrology has a long history in traditional Chinese medicine (TCM) discussions concerning andropathies, and documentation of relevant therapeutic methods abound in the ancient literature on TCM. Integrated treatment combining TCM and Western medicine has seen both broad and in-depth development, with formidable status in the field of modern andrology in China. This article attempts to demonstrate the unique advantage of integrated treatment in the therapy of andropathies through a review of the ancient literature on andrology in the field of TCM and on the integrative treatment of prostatic diseases, sexual dysfunction, male infertility and late-onset hypogonadism. There is a need for the advancement of a medical theory that integrates TCM and Western medicine practices to create a new therapeutic system with standardized therapeutic and evaluative protocols for diseases involving male sexual health.
\end{abstract}

Asian Journal of Andrology (2011) 13, 592-595; doi:10.1038/aja.2010.127; published online 6 June 2011

Keywords: andrology; integrated medicine; traditional Chinese medicine; Western medicine

\section{INTRODUCTION}

Andrology in both traditional and integrated medicine has been particularly widely developed and applied in China. Andrology, as a formal field of medicine in modern-day China, was started in the late 1980s. Traditional Chinese medicine (TCM) and integrated medicine played important roles in the practices of Chinese andrology soon thereafter.

China's history reflects an emphasis on males in society and stresses men's sex, as well as diet, as being essential and indispensable, as depicted by the classical saying, 'The desire for food and sex is a part of human nature'. Therapeutic knowledge about andropathies was introduced via bamboo slips that were unearthed from Mawangdui Han tomb, which reflects a long history of TCM in the diagnosis and treatment of andropathies. The recording of sexual techniques can be found in the He Yin Yang (integration of Yin and Yang), the Yu Fang Mi Yao \& Mi Jue (Recipe for Sexual Intercourse) and the Qian Jin Yao Fang, as well as in Buddhist and Taoist documents, poems and songs. The early use of prescriptions, Qigong (a kind of exercise for staying healthy, developed in ancient China) and therapeutic guidelines can be found in many works of ancient literature.

Ancient literature in TCM mainly described sex as it pertains to subjective sensation and the actual performance of the body in sexual life. Moreover, TCM summarized instructions, methods and matters to direct sexual activity, including 'ten motions', 'seven impairments' and 'eight benefits', which asserted that the sexual life should be moderate, that men may not marry before 30 years of age and that women may not marry before 20 years of age. Even now, these theories have a basis in science.

TCM also introduces a theory surrounding the retention of urine and dysuria. Additionally, TCM discussions on hematuria with turbid discharge and turbid urine have important significance for the treatment of chronic prostatitis today. Essentially, figures from ancient history offered an introduction and an accumulation of experience in the diagnosis and treatment of several major diseases in the field of andrology. This influence has practical significance for guiding diagnosis and treatment today and is also very important for the field of integrated medicine with respect to disease diagnosis, clinical trials and scientific research.

Historical practices in andrology enrich present-day therapies in the field. Integrated andrological treatment further improves and contributes to traditional treatments in the aspect of syndrome differentiation, and progress in Western medicine related to disease differentiation allows for improved clinical differentiation of andropathies that can be used more consistently in clinical practice. The modern scientific method has been especially valuable in researching TCM treatments of male sexual health and has enabled TCM to feature Chinese characteristics in a more modern, scientific and practical manner. This process reveals the nature of modern medicine, which integrates traditional and Western techniques.

Andrology in TCM, especially in integrated medicine, offers a unique advantage in the treatment of male sexual health.

\section{PROSTATIC DISEASES}

Chronic prostatitis

In the theory of TCM, chronic prostatitis is characterized by hematuria and hematuria with turbid urine. Such classification does not meet the practical demands involved in clinical treatment. 
Chronic prostatitis, particularly $\mathrm{III}_{\mathrm{b}}$ (pelvic pain syndrome) is part of a group of disorders involved in chronic pelvic pain syndrome and lacks experimental objective indicators or microbiological indicators. Integrated medicine aims at adequate relief of symptoms of chronic prostatitis. TCM adopts the principle of treatment based on syndrome differentiation, and Chinese drugs are dispensed for a specific symptom. Emphasis is placed on eliminating the disorder and improving the quality of life. For example, in TCM, if pain is caused by inflammation and oedema, the diagnosis is damp-heat lower jiao, which means the damp and heat accumulated in the lower limb. In TCM, damp and heat are two of the pathogenic factors. Lower jiao is a part of sanjiao, which is an organ name. If pain is caused by localized, pelvic muscle spasms, the problem is liver qi obstruction. If pain is caused by obstruction of prostatic flow due to congestion and inflammatory node compression, the diagnosis is blood stasis due to the sluggishness of $q i$ ( $q i$ is a fundamental substance that maintains the normal vital activities of the human body in TCM and mainly provides motive force). Either weakness in propelling blood due to qi deficiency or retardation of $q i$ circulation may cause disorders of blood circulation or even stagnation of blood. If the pain is caused by anxiety, the problem is unconsciousness. The disease may be treated in principle by a prescription to remove damp-heat from lower jiao, which will disperse the stagnated liver $q i$. This process means relieving the depressed liver, regulating $q i$ flow and promoting blood circulation by correcting blood stasis. Therefore, the treatment of chronic prostatitis using integrated traditional and Western medicine has obvious advantages and can greatly promote and enrich traditional clinical treatment of chronic prostatitis. Recently, a clinical trial implemented in Guangdong Province used the alpha antagonist Cardura XL in the control group and the Chinese patented medicinal compound Xuanju in the experimental group to treat chronic prostatitis due to a deficiency of kidney-yang. Diagnosis of kidney disease in TCM is not the same as renal insufficiency in Western medicine. A total of 120 cases were randomly selected for administration of the control medicine. Both International Index of Prostatic Symptoms Score and International Index of Erectile Function-5 scoring revealed that the Chinese patented medicine had a stronger effect than did Cardura XL. ${ }^{1}$

An analysis of more than 5000 evidence-based medical studies from the past 10 years offers proof that TCM produces positive effects in the treatment of chronic prostatitis. The methods of removing damp-heat from lower jiao for lower urinary tract symptoms and promoting blood circulation to treat stasis in cases of dysuria and pelvic pain are used frequently and effectively. The evidence for this recommendation is classified as grade $\mathrm{A}^{2}$ (grade $\mathrm{A}$ : at least one random control clinical experiment is necessary for publication. grade B: a clinical study related to the subject and completed with good results is required, without the need for a randomized controlled clinical experiment. grade $\mathrm{C}$ : reports or opinions and/or clinical experience from expert committees are required, but not direct and high-quality clinical studies). Additionally, enemas in TCM and localized injections are also effective in treating chronic prostatitis. The evidence for this recommendation is classified as grade A, but this option is not recommended as a front-line therapy unless other treatments are ineffective. Ba Zhen San and Long Dan Xie Gan Wan (traditional herbal formulas) may be used to remove damp-heat accumulation in lower jiao and for urethral stimulation; the evidence for this recommendation is classified as grade B. ${ }^{3,4}$ Tao Ren $\mathrm{Si}$ Wu (traditional herbal) formulas in combination with Long Dan Xie Gan (traditional herbal formula) and Gui Zhi Fu Ling Wan (traditional herbal formula) may be used to correct damp-heat stasis and localized referred pain; the evidence for this recommendation is classified as grade $\mathrm{B} .{ }^{5}$ Zuo Gui Wan (traditional herbal formula) may be used to improve both liver- and kidney-yin insufficiencies and poor sexual function; the evidence for this recommendation is classified as grade C. Chai Hu Shu Gan San (traditional herbal formula) and Xiao Yao San (traditional herbal formula) may be used to treat liver qi stasis, anxiety and nerves; the evidence for this recommendation is classified as grade B. ${ }^{6}$ A retention enema may be used as an auxiliary method to treat chronic pelvic pain syndrome; the evidence for this recommendation is classified as grade $B{ }^{7}$ Shuan Ji Na Gang (a kind of suppository) carries a recommendation that is grade A. ${ }^{8}$ Acupuncture may also be used to treat prostate pain syndrome; the evidence for this recommendation is classified as grade B. ${ }^{9}$

Clinically, among some patients with prostatitis, the prostatic fluid cannot be relieved because of the dense prostatic fluid blocking the prostatic urethra. The Chinese medicine technique of warming the kidneys can increase semen volume and help the flow of prostatic fluid, thus relieving and eliminating localized pain caused by stagnation of prostatic fluid. In conclusion, removal of damp-heat from the lower jiao and the promotion of blood circulation are used frequently and efficiently to treat chronic prostatitis.

\section{Prostatic hyperplasia}

Benign prostatic hyperplasia $(\mathrm{BPH})$ is very common among middleaged and elderly male patients. The condition is caused, according to Western medical theory, by increased age and the disordered metabolism of male sex hormones. According to TCM theory, BPH is caused by renal insufficiency and falls in the category of urinary retention. According to integrated medicine theory, $\mathrm{BPH}$ is a representation within the prostate of the degeneration of organs and tissues as one ages; the condition represents the overall ageing process and a depression of overall metabolism and organ function, including depressed function of the detrusor muscle. Simply restricting testosterone from transforming into dihydrotestosterone or nourishing the kidneys could not obtain the therapeutic effects that we expected in the treatment of BPH. Through many years of clinical practice, we found that BPH patients suffered from dysfunction of the zang-fu organs, depression of whole-body metabolism, a deficiency of $q i$, stagnant blood, a deficiency of kidney-yang, mental lethargy, tiredness, cold extremities, prostatic hyperadenosis, increased pressure in the distal urethra and depressed detrusor function. The symptoms included dysuria, nocturnal frequency and long voidings of clear urine. To target these symptoms, Jia presented a new therapy for supplementing qi, activating blood circulation, nourishing the kidneys and removing damp-heat. He developed a prescription medication that passed a national clinical study for novel medicines and a 15month randomized, blind, controlled clinical trial. The results from the clinical trial proved that the medication was effective. After 6 weeks of administration, maximum urine flow was increased by $3.1 \mathrm{ml} \mathrm{s}^{-1}$, lower urinary tract symptoms were remarkably improved, the International Index of Prostatic Symptoms Score was reduced, quality of life was improved and the prostate volume had decreased to some extent. Importantly, we launched research on the mechanism of BPH treatment using TCM with financing from five natural science foundations. It is found that the new medication can inhibit $5-\alpha$ reductase, block $\alpha$-receptor, downregulate prostatic tissue bFGF and TGF- $\beta$ and upregulate nitric oxide synthase. Additionally, BPH treatment can strengthen the detrusor muscle. ${ }^{10-12}$

The effects of Chinese patented medicines and vegetable preparations on the clinical treatment of $\mathrm{BPH}$ have been recognized by domestic and international clinical physicians and have gradually 
become the major therapeutic option for the clinical treatment of $\mathrm{BPH}$.

\section{SEXUAL DYSFUNCTION}

Sexual dysfunction, especially erectile dysfunction (ED), is a common symptom of many diseases. The cause of sexual dysfunction is complicated and may be divided into three categories: psychogenic causes, organic causes (including endogenous, vascular and drug causes) and mixed causes. Currently, research focuses on the dysfunction of endothelial cells of the cavernous body of the penis and disordered release of NO. To date, several phosphodiesterase type- 5 inhibitors have been developed. However, the goal of most doctors and patients is not for the patient to have an occasional erection but to have a natural and harmonious sexual life. TCM achieves better overall regulation, especially with regard to ED patients' anxiety, fatigability, changing hormonal levels, insomnia and gastroparesis. Medicine to invigorate $q i$ can enhance physical fitness, and medicine to warm the kidneys can regulate sex hormones, increase sexual drive, invigorate the spleen, regulate the stomach and improve the overall situation. Medicine used for a stagnated liver provides tranquilisation and helps stabilize the mind, which can improve mental processes and emotional wellness. This treatment can not only increase the effects but also improves the patient's overall condition and quality of life.

In the past, traditional treatments based on syndrome differentiation (an overall analysis of signs and symptoms) placed importance on the kidneys and liver. However, using the concepts of integrated medicine, sexual dysfunction, especially ED with premature ejaculation, should be treated concurrently based on syndrome differentiation of the heart. This approach does not conflict with the concept of TCM that the heart controls mental activities, blood circulation and eroticism. Concurrent treatment of the heart and kidneys can coordinate these organs. Thus, the concept of integrated medicine offers a perfect, traditional treatment for erectile dysfunction and premature ejaculation.

According to the latest pharmacological research on TCM, many Chinese medicines (e.g., ginseng, epimedium and pilose antler) function as the male sex hormone. According to domestic and international research, ginsenoside and red ginseng extracts can stimulate penis tissue to produce $\mathrm{NO}$ and phosphodiesterase type-5 inhibitors. Additionally, ginsenoside and red ginseng extracts can also regulate the function of sex glands and increase semen volume to reinforce sexuality. Epimedium and lycium berry can inhibit nitric oxide synthase and are helpful for improving endothelial cell function in the penis and promoting the formation of NO. ${ }^{13}$ Research has shown that Chai Hu Shu Gan San can increase the duration of erection in the male rat and can increase NO content in penis tissue. Medicine that promotes blood circulation, such as Tao Ren Si Wu and Jin Kui Shen Qi Wan, can help to regain an erection that was lost or achieve a repeat erection. Therefore, the treatment of ED with TCM has practical effects and is supported by scientific research.

Overall, TCM treatment for sexual dysfunction can not only increase the effects of simultaneous treatments but also improve the patient's overall condition and quality of life.

\section{MALE INFERTILITY}

Numerous studies have been conducted on the traditional treatment of infertility. In the past 10 years, more than 500 papers have been published on traditional treatment methods for oligospermia and asthenospermia. All of these studies reflect the varying degrees of effect that TCM has had on the treatment of oligospermia and asthenospermia.
However, from the perspective of integrated medicine, better effects are achieved if the medicine can promote blood circulation in the testes and invigorate qi. Therefore, the following therapeutic concept has been proposed: treating oligospermia by promoting blood circulation and tonifying the kidneys and treating asthenospermia by warming the kidneys and supplementing qi. Statistical data from August 2009 through July 2010 show that after such treatment, semen density and activity increased among 465 cases; the total effective rate reached $52.6 \%$ (unpublished data). This therapy is referred to as Yi Jing Fang (herbal formula) and is now used for clinical therapy.

The investigation on the mechanism of Yi Jing Fang involves: use of an adenine method to produce a male Wistar rat model of infertility with kidney-yang insufficiency. Cyclophosphamide was then injected into the male rat's abdominal cavity at a dose of $60 \mathrm{mg}$ per $\mathrm{kg}$, once daily for 5 days. Then, a concentrated Yi Jing Fang solution was administered via the stomach once daily for 34 days. The following measurements were taken: semen apoptosis rate, mitochondrial function, intracellular $\mathrm{Ca}^{2+}$ concentration and expression of the CatSperl gene coding for the calcium channel specific to semen. The results showed that Yi Jing Fang significantly strengthened expression of the CatSper1 gene, improved semen mitochondrial function, increased intracellular $\mathrm{Ca}^{2+}$ concentrations, strengthened semen activity and remarkably reduced semen apoptosis. ${ }^{14}$

We conducted a retrospective study with 207 male patients at the Department of Andrology of Guang'anmen Hospital from 2006 to 2008. We evaluated the density, activity and motility rate of the semen before and after treatment. The results showed that after the treatment for oligospermia and asthenospermia with Yi Jing Fang, the semen density at three different levels and the total semen density were remarkably increased $(P<0.05)$, while semen activity and motility rate were also greatly improved $(P<0.05) .{ }^{15}$

Generally, tonification of the kidney and promotion of the blood circulation are used frequently and efficiently to treat male infertility.

\section{LATE-ONSET HYPOGONADISM (LOH)}

In Western medicine, $\mathrm{LOH}$ is considered to occur due to a deficiency of male hormones, but the deficiency in the kidney is believed to be the culprit in TCM. In the theory of integrated medicine, LOH is a syndrome that arises from the depression of overall function with age and cannot be cured solely by supplementing male hormones and tonifying the kidneys. Rather, the condition must be treated based on the principle of overall health care and the postponement of ageing.

In Western medicine, $\mathrm{LOH}$ is caused by the depression of free testosterone. In the theory of traditional treatment based on syndrome differentiation, LOH occurs after a breakdown in coordination between the heart and the kidneys, deficiencies of the spleen and kidney-yang, deficiencies of the liver and kidney-yin and deficiencies of the kidney-yin and -yang. As one ages, endocrine function of the pituitary gland and sex glands becomes disordered, as do the distribution and function of male hormone receptors in certain tissues and organs. This problem occurs due to a depression of overall function. The overproduction and accumulation of free radicals, upregulation of apoptosis and decreased self-repairing capacity cannot be relieved solely with supplementation of male hormones. TCM can invigorate the spleen and stomach, thus benefiting the body. Specifically, warm yang can tonify kidneys to benefit the body before birth, remove toxins, invigorate $q i$ and promote blood circulation to lengthen life. ${ }^{16}$ This therapy can remove free radicals, regulate blood fat, improve cardiocerebral blood flow, improve function of the digestive, respiratory and endocrine systems, and regulate the body in every aspect. 
This background provides the thought and starting point of integrated medicine with respect to the knowledge and treatment of $\mathrm{LOH}$.

$\mathrm{LOH}$ is frequently found in elderly and middle-aged male patients. LOH develops through a specific process, including disordered work and rest, excessive stress, depression, hyperphagia and delayed treatment for illness. All of these phenomena can accelerate the onset of LOH. ${ }^{17}$ The terms 'fatigue syndrome' and 'death from overwork' should carry great importance.

The common approach to the prevention of this process based on the principle of integrated disease and syndrome differentiation is to promptly and effectively disperse the stagnated liver $q i$, invigorate the spleen, replenish qi, reinforce the kidneys, nourish the blood and tranquilize the mind.

\section{PROSPECTS}

Differentiation is a unique feature of TCM therapy in the context of men's health

Clinical practice in TCM has been an exemplary case of customized treatment for over a thousand years. Holism and differentiation epitomize the essence of TCM. Treatment differentiation refers to customized clinical practice based on the standard diagnosis protocol, treating patients with the same disease with different combinations of herbs depending on timing, location and the individual patient's condition. Such differentiated treatment, as a unique feature of TCM, combines diagnostic differentiation and corresponding treatment. Differentiation hinges on four interrelated components integral to the diagnostic process: reviewing the patient's case history, identifying possible causes, defining symptom characteristics and drawing initial conclusions about the nature of the disease. The corresponding treatment, weighing the pros and cons of the different options, then draws up the therapeutic solutions according to TCM theories. Treatment of diseases men's health needs to follow a clear identification and definition of the disease and the syndrome. Patients with the same disease and different syndromes should be treated by different herbs, while patients with different diseases and the same syndrome should be treated with the same herbs. The TCM teaching on 'the same treatment for the same syndrome' dovetails well with the wisdom from Western medicine: 'the same treatment for the same therapeutic target'. ${ }^{18}$

Establishment of an integrated medical theory and new diagnostic and treatment system for andropathies

Andropathies, including ED, infertility, BPH, chronic pelvic pain syndrome and $\mathrm{LOH}$, are not caused by a single causa morbi but are related to age, the environment, and social and psychological factors. Therefore, a therapeutic approach that addresses overall regulation, integrates disease and syndrome differentiation and reflects individualized treatment is required. Integrating symptomatic treatment, enhancing the body and improving one's overall quality of life serve as excellent guidelines.

With respect to the grave study of disease aetiology and the exploration of new therapeutic methods and guidelines, the integration of traditional and Western medicine will serve to better reflect their respective advantages and enhance diagnostic and therapeutic approaches to embody the characteristics of Chinese andrology.

These goals can be ascertained by fully focusing on TCM and establishing a research system to integrate traditional and Western medicine related to major issues in the development of medical science.

Using traditional Chinese theories of medicine to understand disease allows for promising breakthroughs in clinical treatment. This strategy may allow for the development of new therapeutic thought if pharmacological research achievements in TCM modernisation can be prioritized in treatment with Chinese medicine.

TCM asserts the homology of medicine and diet. We can accomplish more with andrology through integrated traditional and Western treatment if we summarize and develop new preventive and therapeutic theories, prescriptions, diets and health care methods with theories from TCM related to preventive treatment.

\section{Establishment of standardized therapeutic and evaluation systems} for integrated medicine

A standard therapeutic and evaluation system should be established with reference to the advanced content of diagnostic, therapeutic and evaluation standards of modern medicine. These new systems need to concurrently reflect the concepts and advantages of TCM and integrated medicine. Furthermore, these standardized therapeutic and evaluation systems should be manageable, easy to use, and fully operational and will combine the advantages of both TCM and Western medicine.

In the past thousands of years, TCM has accumulated abundant experiences in treating men's health diseases. In recent decades, TCM practitioners have made sustained efforts in wide scope and in-depth clinical research on many aspects of men's health, including research on the effective agents, evolution of the patterns of syndromes and normalized study of syndromes. Many useful new theories and therapeutic methods have been advanced based on empirical research to help facilitate clinical practice. We believe that the integrated treatment combining TCM and Western medicine will play more important roles in the treatment of men's health diseases.

1 Zhu XW, Chen ZD, Yao XL, Fang JJ, Du CJ et al. Chinese patented medicinal compound Xuanju for the treatment of chronic prostatitis III. Chin J Androl 2010; 24 (8): 56-7.

2 Qiu MX, Xiong GB, Zhou SY, Wang D, Shao JD et al. Qingrelishi-category Chinese medicine for chronic prostatitis: a systematic review. Zhonghua Nan Ke Xue 2007; 13: 370-7.

3 Shen J, Xie X. Effects of Bazhengsan on IL-1 and TNF- $\alpha$ in EPS of the patients with type II chronic prostatitis. J Guiyang College Tradit Chin Med 2008; 30 (3): 35-7.

4 Pang YR, Mo QP. Bazhengsan for 86 cases of damp-heat type chronic prostatitis. J North China Coal Med College 2009; 11: 353-4.

5 The Professional Committee on Urinary Surgery of National Integrative Chinese and Western Medicine Academy. Guidance for diagnosis and treatment of chronic prostatitis by integrative medicine. Chin J Integr Tradition West Med 2007; 27: 1052-6.

6 Liu HG, Ma WJ. Efficacy of Chaihushugansan in the treatment of chronic nonbacterial prostatitis. Guangxi J Tradition Chin Med 2008; 31: 211-2.

7 Zhou SY, Wang L, Xiong GB. Clinical observation on treatment of chronic prostatitis syndrome by Wuwei Xiaodu decoction. J Sichuan Tradit Chin Med2006; 24 (6): 50-1.

8 Xu G, Lu J, Tang XD, Bo JJ, Wang YX et al. Efficacy and safety of prostant in the treatment of chronic prostatitis: a multi-center, randomized, double-blinded, placebo-controlled trail. Chin J Urol 2002; 23: 296-9.

9 Huang YJ, Fan XH, Du M. Acupuncture therapy for 42 cases of chronic prostatitis. $J$ Clin Acupunct Moxibust 2005; 21 (4): 8-9.

10 Jia JM, Li YZ, Cui YG, Zhang GY. Effects of Xiaolong Tongbi on T, E2, DHT, AR and $5 \alpha-$ reductase in canine prostate tissue. Bull Med Res 1998; 27 (9): 39-41.

$11 \mathrm{Jia}$ JM, Ma CT, Wang LY, Bi ZC. Experimental study on effect of Xiaolong Tongbi on nitric oxide synthase and epithelial cell apoptosis in prostate of rats. Chin J Integr Tradit West Med 2002; 22: 196-9.

12 Ma WG, Jia JM, Jiao YZ, Wang JH, Luo SB et al. Longbixiao capsule reduces expressions of TGF- $\beta 1$ and smoothelin in human prostatic stromal cells cultured in vitro. Zhonghua Nan Ke Xue 2008; 14: 466-70.

13 Fu J, Qiao L, Jing TY, Lin GT, Wang YY et al. Effect of icarrin on cGMP levels in penile corous cavernosum of rabbit. Chin Pharmacol Bull 2002; 18: 430-3.

14 Wang JH, Chen D, Zhou JG, Cao YG, Jia JM. Construction of the male rat model of adenine-induced infertility with kidney-yang deficiency. Zhonghua Nan Ke Xue 2008; 14: 556-70.

15 Luo SB, Jia JM, Ma WG, Dong JC, Jiao YZ. Yijing prescription for 207 sperm weakness patients. Shaanxi J Tradit Chin Med 2010; 31: 410-3.

16 Zheng XF, Li P. Clinical thinking on acupuncture-moxibustion treatment of partial androgen deficiency in aging men. Shanghai J Acupunct Moxibust 2006; 25 (4): 28-9.

17 Fan XB, Huang YF. Testosterone undecanoate for late-onset hypogonadism: an update. Zhonghua Nan Ke Xue 2010; 16: 68-71.

$18 \mathrm{Li} \mathrm{J}$. Thinking on syndrome differentiation treatment and personalized therapy for tumor. J Chin Integr Med 2009; 7: 306-8. 